\title{
Chronic liquid fructose-, but not glucose-, supplementation selectively induces visceral adipose tissue leptin resistance and hypertrophy in female Sprague-Dawley rats
}

Gemma Sangüesa ${ }^{1,2}$, Núria Roglans ${ }^{1,2,3}$, José Carlos Montañés ${ }^{1}$, Miguel Baena ${ }^{1,2}$, Ana Magdalena Velázquez $^{1}$, Rosa María Sánchez ${ }^{1,2,3}$, Marta Alegret ${ }^{1,2,3^{*}}$, Juan Carlos Laguna ${ }^{1,2,3 *}$

${ }^{1}$ Department of Pharmacology, Toxicology and Therapeutic Chemistry, School of Pharmacy and Food Science, University of Barcelona, ${ }^{2}$ Institute of Biomedicine University of Barcelona, and ${ }^{3}$ Centro de Investigación Biomédica en Red de Fisiopatología de la Obesidad y Nutrición (CIBERObn), Spain.

*These authors contributed equally to this work

Keywords: hypothalamus, liver, SOCS3, sugar sweetened beverages,

Corresponding authors: Marta Alegret (ORCID \# 0000-0002-5652-8651, alegret@ub.edu) and Juan Carlos Laguna Egea (ORCID \# 0000-0002-7082-0704, jclagunae@ub.edu), Department of Pharmacology, Toxicology and Therapeutic Chemistry, School of Pharmacy and Food Science, University of Barcelona, Avda. Joan XXIII 27-31, Barcelona 08028, Spain, tel: 34934024530

Abbreviations: AGRP (agouti-related protein), AKT (V-akt murine thymoma viral oncogene homolog2), AMPK (AMP-activated protein kinase), ATGL (acyl-triacylglycerol-lipase), CART (cocaineamphetamine-related peptide), ERK1/2 (extracellular signal-regulated kinase 1/2), FASN (fatty acid synthase), HSL (hormone-sensitive lipase), IL-6 (interleukin-6) IRE1 (inositol requiring enzyme 1), NEFA (non-esterified fatty acid), NPY (neuropeptide Y), ObRb (long form of the leptin receptor), PKA (protein kinase A) POMC (proopiomelanocortin), SCD1 (stearoyl-CoA desaturase), SOCS3 (suppressor of cytokine signaling 3), SSB (sugar-sweetened beverages), STAT3 (signal transducer and activation of transcription 3), TNF $\alpha$ (tumor necrosis factor alpha), UPR (Unfolded Protein Response), vWAT (visceral white adipose tissue), 


\begin{abstract}
Scope: We studied the effect on leptin signaling in liver, hypothalamus and visceral white adipose tissue (vWAT) of chronic supplementation with simple-sugar solutions, designed to mimic the temporal pattern of consumption by humans. Methods and Results: Solutions of fructose or glucose were isocalorically supplemented (7 months) in female Sprague-Dawley rats consuming ad libitum rodent chow. After sacrifice, plasma and tissue samples (liver, hypothalamus and vWAT) were collected. Zoometric parameters, plasma analytes and the tissue expression and activity of markers of leptin signaling were determined by biochemical and molecular biological methods. The two sugars caused different types of adiposopathy. Both sugars induced increases in plasma non-esterified fatty acids, and leptin resistance in liver and hypothalamus. Only fructose-supplemented rats showed hyperleptinemia, and increased body weight due to a hypertrophy of vWAT, with no signs of leptin-mediated lipolysis. Glucose-supplemented rats showed no significant changes in these parameters but presented elevated plasma adiponectin concentrations, lipolysis and inflammatory markers in vWAT, indicating a shift to a non-expandable adipose tissue phenotype. Conclusion: Chronic consumption of fructose places a greater burden on metabolic homeostasis than equivalent consumption of glucose, inducing hyperleptinemia, generalized leptin resistance, and increased body weight due to expanded, hypertrophic vWAT.
\end{abstract}




\section{Introduction}

High and sustained levels of consumption of sugar-sweetened beverages (SSB) is a key dietary trait in the many phenotypic factors involved in the current epidemics of obesity and related cardiometabolic pathologies. Fructose and glucose are the simple sugars present in SSB, in the form of either sucrose or high fructose corn syrup. The excess of calories provided by sustained SSB intake is definitely a clear contributor to the metabolic alterations associated with SSB consumption by human populations, but there is also a lively ongoing debate concerning whether the specific metabolism of fructose has additional deleterious effects [1, 2][3]. In this sense, Stanhope et al [4] has clearly shown in a randomized clinical trial conducted for 10 weeks on 32 overweight subjects that, at similar caloric intake (25\% of total energy consumed), fructose alters lipid and glucose homeostasis more intensely than glucose.

Supplementation of rats with liquid fructose is a tried and tested experimental model to study the impact of fructose consumption on lipid and glucose metabolism [5]. However, rats are often supplemented only for short periods of time (2-8 weeks) [6-12], a situation that does not accurately mimic chronic SSB consumption in human populations. In order to obtain new data from an experimental model mimicking chronic human consumption of fructose-containing beverages, here we supplemented female SpragueDawley rats with liquid fructose and glucose solutions, matching the quantity of calories they provide daily, for a 7-month period. Considering that one rat month is approximately equivalent to three human years [13], this supplementation period is roughly equivalent to 15-20 years of human life. Using this experimental model we have previously shown that, despite an increase in daily calorie intake of more than $50 \%$ with respect to control animals, liver homeostatic mechanisms reverts the liver fat deposition previously observed in fructose-supplemented rats over shorter periods of time, probably by a specific activation of the inositol requiring enzyme 1 (IRE1) branch of the Unfolded Protein Response (UPR) [14]. However, rats supplemented with fructose for seven months clearly showed extra-hepatic impaired insulin signaling and glucose intolerance [15], two metabolic alterations already detected in rats supplemented with fructose for two months $[9,12]$. Moreover, impaired insulin signaling in cortex tissue from these rats was related to a cognitive deficit, as assessed by the novel object recognition test [15].

In the present work, we show that female Sprague-Dawley rats supplemented for seven month with a liquid simple-sugar (fructose or glucose) show: hyperleptinemia and liver leptin resistance, two metabolic alterations already detected over shorter periods of fructose supplementation [7, 10]; hypothalamic leptin resistance; and, only in the case of fructose-supplemented rats, visceral white adipose tissue (vWAT) leptin resistance that resulted in hypertrophy of adipocytes and increased body weight.

\section{Materials and Methods}

\subsection{Animals}

Female Sprague-Dawley rats were obtained from Charles River (Barcelona, Spain), and were maintained under conditions of constant humidity (40-60\%) and temperature $\left(20-24^{\circ} \mathrm{C}\right)$ with a light/dark cycle of 12 hours. Procedures were conducted in accordance with the guidelines established by the University of Barcelona's Bioethics Committee (Autonomous Government of Catalonia Act 5/1995, of July 21). All experimental procedures involving animals were approved by the University of Barcelona's Animal Experimentation Ethics Committee (approval No. 7912).

\subsection{Dosage Regimen}

24 adult rats (aged eight weeks) were randomly assigned to either a control group (no supplementary sugar, $n=8)$, a fructose-supplemented group $(10 \% \mathrm{w} / \mathrm{v}$ in drinking water $)(\mathrm{n}=8)$ or a group supplemented with a glucose solution prepared to match the amount of calories ingested by the fructose group $(n=8)$, for seven months (28 weeks), in order to mimic chronic consumption of SSB by humans. The animals received 2014 Teklad rodent maintenance diet (Envigo, Barcelona, Spain). Throughout the treatment, the 
amount of solid food ingestion was controlled weekly and liquid consumption three times a week. Body weight was assessed every two weeks. Each rat consumed between 3.3-3.9 g of simple sugar per Kg and day, while high levels of simple sugar consumption in humans can reach up to $2 \mathrm{~g}$ per $\mathrm{Kg}$ and day [16].

\subsection{Sample preparation}

At the end of treatment, fasting triglycerides and glucose levels were measured in blood samples using an Accutrend ${ }^{\circledR}$ Plus system glucometer (Cobas, Roche Farma, Barcelona, Spain), with blood samples obtained from the tail vein. Then, rats were anesthetized with ketamine/xylazine ( $9 \mathrm{mg}$ and $40 \mu \mathrm{g} / 100 \mathrm{~g}$ body weight, respectively) and blood was collected by cardiac puncture. Plasma was prepared by centrifugation at $1000 \mathrm{xg}$ for $10 \mathrm{~min}$ at room temperature. The rats were then euthanized by exsanguination and visceral white adipose tissue (vWAT; including retroperitoneal, perirenal and perigonadal adipose tissue) was obtained and weighed. Brains were extracted and the frontal cortex, hippocampus and hypothalamus were separated. All the samples were frozen in liquid nitrogen, and stored at $-80^{\circ} \mathrm{C}$ until needed.

\subsection{Blood and plasma parameters}

Concentration of leptin, adiponectin and insulin in plasma were determined using specific enzyme-linked immunosorbent assay kits (Millipore, Billerica, MA, USA). Plasma NEFA levels were determined using a colorimetric assay kit from Bioo Scientific (Austin, TX, USA).

\subsection{Histological Studies}

Paraffin-embedded adipocytes sections were stained with hematoxylin and eosin to assess adipocyte size at BioBanc (Banc de tumors-IDIBAPS, Barcelona, Spain). Images were acquired with an Olympus BX51 microscope. The area and number of adipocytes were calculated with the ImageJ software (NIH, USA) from at least 30 images from each sample $(n=5)$. Analysis was performed with a semiautomated methods, as described by Parlee et al [17].

\subsection{RNA preparation and analysis}

Total RNA was isolated with the TRIzol Reagent (Invitrogen, Carlsbad, CA, USA), in accordance with the manufacturer's instructions. RNA concentration and purity were measured spectrophotometrically using the NanoDrop ${ }^{\circledR}$ ND-1000 Spectrophotometer (Thermo Scientific). The ratios of absorbance at 260/230 and 260/280 were used as indicators of RNA purity. Specific mRNAs were assessed by real-time reverse transcription polymerase chain reaction (RT-PCR) using SYBR Green PCR Master Mix, specific primers and the Applied Biosystems StepOnePlus Sequence Detection System (Applied Biosystems, Foster City, CA, USA). TATA box binding protein ( $T b p)$ was used as an internal control. Primer sequences and PCR product length are listed in Supporting Information Table S1.

\subsection{Preparation of protein extracts}

Total protein extracts from white adipose tissue, frontal cortex, hippocampus and hypothalamus were micronized through freezing with liquid nitrogen and grinding with a mortar. Then, RIPA lysis buffer with proteases, phosphatases and acetylases inhibitors (50 mM Tris- $\mathrm{HCl} \mathrm{pH}=8,150 \mathrm{mM} \mathrm{NaCl}, 1 \%$ Igepal, 10mM NaF, 1 mM EDTA, 1mM EGTA, 2 mM Nappi, 1mM PMSF, $2 \mu \mathrm{g} / \mathrm{mL}$ leupeptin, $2 \mu \mathrm{g} / \mathrm{mL}$ aprotinin, $1 \mathrm{mM} \mathrm{Na} \mathrm{VO}_{4}, 10 \mathrm{mM} \mathrm{NaM}, 1 \mu \mathrm{M}$ TSA) were added to micronized tissue and homogenized for $1,5 \mathrm{~h}$ at $4^{\circ} \mathrm{C}$. Samples were centrifuged at $15,000 \times \mathrm{g}$ for $15 \mathrm{~min}$ at $4^{\circ} \mathrm{C}$ and supernatant was collected. Total protein concentration was determined by the Bradford method [18].

\subsection{Western blot analysis}



Mol. Nutr. Food Res.
G. Sangüesa et al.
Chronic liquid fructose-, but..

Different protein extracts $(20-30 \mu \mathrm{g})$ from tissues samples were subjected to SDS-polyacrylamide gel electrophoresis. Proteins were then transferred to Immobilon polyvinylidene difluoride transfer membranes (Millipore, Billerica, MA, USA), blocked for $1 \mathrm{~h}$ at room temperature with $5 \%$ non-fat milk solution in Tris-buffered saline (TBS) with $0.1 \%$ Tween-20, and incubated as described previously [14]. Detection was performed using the ECL chemiluminescence kit for HRP (Pierce ECL Western Blotting Substrate, Rockford, IL, USA). To confirm the uniformity of protein loading, blots were incubated with $\beta$-actin or $\beta$-tubulin antibody (Sigma-Aldrich, St. Louis, MO, USA), which was used as the normalization control for Western blot analysis. Primary antibodies for total (\#9272) and phosphorylated Akt (\#4058), total (\#2532) and phosphorylated AMPK (\#2535), Tyr ${ }^{705}$ phosphorylated STAT3 (\#9131) and total (\#4107) and phosphorylated HSL (\#4126) were supplied by Cell Signaling (Danvers, MA, USA). The total STAT3 (sc-482), SOCS3 (sc-9023), PKAc (sc- 28315) and total (sc-514302) and phosphorylated ERK1/2 (sc-81492) antibodies were purchased from Santa Cruz Biotechnologies (Dallas, TX, USA).

\subsection{Statistical analysis}

The results are expressed as the mean of $\mathrm{n}$ values \pm standard deviation. Plasma samples were assayed in duplicate. Gaussian distribution of the data was verified using the Kolmogorov-Smirnov normality test, and significant differences were established by one-way ANOVA and Šidák's post-hoc test for selected comparisons. When variance was not homogeneous, a non-parametric test was performed. For the analysis of the difference in the percentage of adipocytes with a surface $<$ or $\geq$ to $8,000 \mu \mathrm{m}^{2}$, a contingence test (Chi-Square) was performed (GraphPad Software V6). The statistical significance level was set at $\mathrm{p} \leq 0.05$.

\section{Results}

\subsection{Chronic liquid simple-sugar supplementation induced hyperleptinemia and liver leptin resistance}

Liver, vWAT, and body weight, solid food and liquid intake, as well as energy consumption of female Sprague-Dawley rats supplemented chronically with liquid fructose and glucose have been reported previously $[14,15]$. In brief, both sugar- supplemented rats increased their total energy intake by $55 \%$. Although both sugar-supplemented groups showed a 37\% reduction in their energy intake from solid feed, this did not compensate the energy provided by the liquid supplementation of sugars and consequently, apparent solid food efficiency, expressed as the ratio between body weight gain and total solid food intake, was similarly increased in fructose- and glucose-supplemented animals $(0.04,0.07$, and 0.07 for control, glucose and fructose groups, respectively). While there was a non-significant tendency for plasma leptin concentrations to increase in glucose-supplemented rats (x1.32 vs control values), in fructose-supplemented rats this reached statistical significance (x2.03 vs control values; Figure 1A). Nevertheless, the consumption of both sugars induced a clear state of leptin resistance in liver tissue, as shown by the marked increase in the liver content of suppressor of cytokine signaling 3 (SOCS3) protein (Figure 1B), while the degree of activation of the signal transducer and activation of transcription 3 (STAT3) protein, ascertained by its phosphorylation at Tyr 727, was not significantly modified (Figure 1C). Moreover, phosphor-AMP- activated protein kinase (AMPK) was also unmodified in samples from fructose-supplemented rats (Figure 1D), despite the marked hyperleptinemia the animals presented.

\subsection{Chronic liquid simple-sugar supplementation selectively induced hypothalamic central leptin resistance}

Simple-sugar supplementation induced a selective state of leptin resistance in the hypothalamus, as shown by the marked increase in the expression of SOCS3 in hypothalamic samples from fructose- and glucosesupplemented animals; while cortex and hippocampus samples showed unmodified SOCS3 expression (Figure 2A). Hypothalamic leptin resistance in samples from simple-sugar supplemented rats was confirmed by the marked increase in the expression of the long form of the leptin receptor $(o b r b)$ and agouti-related protein (agrp), whose expression should be reduced by leptin activity. The expression of 
other genes whose transcription is controlled by leptin, such as the neuropeptide $\mathrm{Y}$ (npy), cocaineamphetamine-related peptide (cart) and proopiomelanocortin (pomc) genes, was unmodified in hypothalamic samples from simple-sugar-supplemented animals (Figure 2B). Moreover, activity of the extracellular signal-regulated kinase $1 / 2$ (ERK1/2) and AMP-activated protein kinase (AMPK), as assessed by the relative abundance of their active phosphorylated forms, was also unchanged in hypothalamic samples from simple-sugar-supplemented animals (Figure 2C).

\subsection{Chronic liquid fructose-, but not liquid glucose-, supplementation induced vWAT leptin resistance}

The effects of insulin on WAT are increased lipogenesis and reduced lipolysis; while the main direct effect of leptin on WAT is increased lipolysis. Plasma from both sugar-supplemented animals showed similar absolute increases in non-esterified fatty acid (NEFA) concentrations (Figure 3A); but when referred to the relative amount of vWAT, only the glucose-supplemented rats showed a significant increase in plasma NEFA (Figure 3B), due to the fact that the fructose-supplemented animals also showed a significant increase in vWAT mass (Figure 3C). Although we detected a decrease in phosphor-V-akt murine thymoma viral oncogene homolog-2 (Akt) in the vWAT samples from both glucose- and fructosesupplemented rats (Figure 3D), only the fructose-supplemented animals showed significant hyperinsulinemia (Figure 3E), implying a more pronounced derangement of insulin signaling in the latter. In agreement with this, only the vWAT samples from the glucose-supplemented rats showed increased expression of genes related to lipogenesis, such as fatty acid synthase fasn or stearoyl-CoA desaturase $s c d 1$ (Figure $3 \mathrm{~F}$ ). With respect to leptin signaling, only the fructose-supplemented animals showed increased SOCS3 expression in vWAT (Figure 4A), with no increase in lipolysis, as shown by the unmodified relative plasma NEFA levels (Figure 3B) and also the lack of increased expression of acyltriacylglycerol-lipase (ATGL) (Figure 4B). Similarly, only visceral adipocytes from the fructosesupplemented rats showed marked hypertrophy (Figure 4C) and a significant shift towards high-surface adipocytes (Figure 4D); a clear signature of adipose tissue leptin resistance. Furthermore, our results seems to indicate that for there to be a significant increase in lipolysis not only is a state of insulin resistance necessary, but so is active leptin signaling, as occurred in the glucose-supplemented animals.

Besides fructose-related hyperleptinemia, we have previously reported [14] that only glucosesupplemented rats show an increased concentration of plasma adiponectin (see Supporting Information Table S2). Although the vWAT expression of leptin and adiponectin genes was not significantly modified by simple-sugar- supplementation (Figure 5A), the plasma leptin:adiponectin ratio, which has been directly correlated with insulin resistance in humans [19], was significantly increased (x1.8 vs control values; Figure 5B) only in fructose-supplemented rats. Furthermore, the activity of markers of increased sympathetic nervous system activity in vWAT, the catalytic subunit of protein kinase A (PKAc) and the amount of phosphor-hormone-sensitive lipase (HSL) were unmodified in fructose-supplemented rats (Figure 5C), while the expression of the $\beta_{3}$-adrenergic receptor was non-significantly up-regulated twofold (Figure 5D). Finally, the expression of F4.80, a marker of tissue macrophages, was unmodified in vWAT from glucose-supplemented rats and significantly reduced in that from fructose-supplemented rats (Figure 5E). This clearly show that despite the massive increase in calorie intake induced by both sugars and the fructose-related adipocyte hypertrophy and increased vWAT mass, there was no increase in the numbers of resident macrophages in vWAT. Despite this, only glucose-supplemented rats showed increases in markers of tissue inflammation, such as tumor necrosis factor alpha (TNF $\alpha$ ) and a similar non-significant tendency in interleukin-6 (IL-6).

\section{Discussion}

We have studied the effect of chronic (over 7 months) supplementing of female Sprague-Dawley rats with liquid solutions of simple sugars, isocaloric glucose and fructose, designed to closely mimic the temporal pattern of consumption of SSB by human populations. Our previous work has shown that, at a similar calorie intake, only fructose selectively induces extrahepatic insulin resistance and mild cognitive impairment, with no signs of excessive fat accumulation in liver [14, 15]. Here, we present data showing 
that under the experimental conditions mentioned above, fructose selectively induces hyperleptinemia and leptin resistance in hypothalamus and vWAT, favoring adipocyte hypertrophy, and an increase in both vWAT and body weight.

We have previously shown that, for short periods of time and when supplemented under isocaloric conditions, fructose induces clear hyperleptinemia and hepatic leptin resistance, but glucose does not [7, 10]. Under chronic supplementation for seven months the hyperleptinemic and hepatic leptin resistance effect of fructose is maintained (Figure 1). Although glucose-supplemented animals showed no significant hyperleptinemia, the small increase in plasma leptin levels induced by glucose supplementation led over time to a similar leptin resistance in liver, as shown by the increase in liver SOCS3 protein content and the lack of STAT3 and ERK1/2 activation [7, 10]. Liver AMPK activation, a downstream marker of leptin signaling [20], was not modified in fructose-supplemented rats, further confirming the production of hepatic leptin-resistance. Although seemingly contradictory, the increase in AMPK activation induced by chronic glucose supplementation (Figure 1D) can be related to the parallel increase in plasma adiponectin levels, that we have previously reported in these animals [14]. Activated AMPK is also a downstream marker of adiponectin signaling in liver tissue [21]. The plasma leptin:adiponectin ratio, which has been directly correlated with insulin resistance in humans [19], was significantly increased only in fructose supplemented rats, which also showed insulin resistance of an extrahepatic origin [15].

Obesity often presents with hyperleptinemia [22] and is associated with impaired transport of leptin across the blood-brain barrier and also with a reduction in the leptin receptor function and signaling in the central nervous system (CNS) [23][24]. Furthermore, hypertriglyceridemia impedes leptin transport to the CNS [25] and triglycerides themselves penetrate the CNS and induce leptin resistance [26]. Chronic supplementation with either simple-sugar in liquid form led to selective leptin resistance in the hypothalamus, but not in the hippocampus or frontal cortex, as assessed by the increased expression of SOCS3, a bona fide marker of tissue leptin resistance [27] [28]. Among other effects, active leptin signaling in the hypothalamus should result in increased and decreased activity, respectively, of ERK1/2 and AMPK, increased anorexigenic gene expression of POMC and CART, and decreased orexigenic gene expression of NPY and AgRP [29]. Hypothalamic samples from simple-sugar-supplemented rats showed no significant changes in the activity of ERK1/2 and AMPK, or the expression of NPY, POMC and CART, while the expression of AgRP was markedly increased by both glucose- and fructosesupplementation. The lack of proper leptin signaling was further confirmed by the marked up-regulation of the expression of the long form of the leptin receptor [30]. As opposed to fructose-supplemented rats, glucose-supplemented rats, despite showing a similar molecular signature of leptin resistance in the hypothalamus, showed no significant increase in accretion of vWAT and, as we have shown previously, no change in plasma triglycerides [14] (see Supporting Information Table S2), indicating that a mild increase of plasma leptin concentration over a substantial period of time probably suffices to induce a clear state of leptin resistance in the hypothalamus, but not in vWAT.

As opposed to the cases of liver and hypothalamic tissue, only liquid fructose-supplemented animals showed the two hallmark modifications related to resistance to leptin signaling in vWAT: a blunted increase in lipolysis in the presence of hyperleptinemia [22] and, consequently, a significant increase in hypertrophic visceral adipocytes [31], resulting in increased body weight and obesity. Increased triglyceride hydrolysis resulting from the action of leptin seems to depend on intact sympathetic activity, not on any direct, autocrine effect of leptin on adipose tissue [32]. In fact, very recently, Bell et al reported the activity of leptin on hypothalamic AgRP neurons, but not POMC neurons, as directly responsible for leptin-induced sympathetic nerve activation to inguinal fat in mice [33]. vWAT from hyperleptinemic fructose-, but not glucose-supplemented rats showed increased expression of the $\beta_{3^{-}}$ adrenergic receptor, and a lack of increased activity of PKA and HSL. Up-regulation of the $\beta_{3}$-adrenergic receptor and a lack of activation of its key transducers, PKA and HSL, clearly indicate a lack of sympathetic stimulation of vWAT in these animals, in accordance with the lack of a leptin-repressive effect on hypothalamic AgRP expression (Figure 2B), which is an indicator of resistance to leptin action 
in hypothalamic AgRP neurons. We do not know why glucose-supplemented rats, although also showing increased de-regulated expression of AgRP in the hypothalamus, showed increased vWAT lipolysis and AGTL expression. However, as we have previously reported for liver metabolism [14], the differential effect of the two simple-sugars on plasma adiponectin and leptin concentrations could be a key factor in explaining the opposing responses of vWAT in glucose- and fructose-supplemented rats, as it is known that adiponectin enhances adipocyte lipid storage [32]. Nevertheless, changes in plasma adiponectin and leptin concentrations after simple sugar supplementation could differ between humans and rats; Rezvani et al. [34] , in a study in overweight men and women with a very similar experimental design to that of Stanhope et al. [4], found that in overweight women, while both sugars, fructose and glucose, increased fasting plasma leptin concentration, only glucose significantly increased fasting plasma adiponectin concentration at the shortest time of supplementation (2 weeks).

Examination of our previous results $[14,15]$ and those presented here on chronic (7-month), liquid simple-sugar isocaloric supplementation of female Spraque-Dawley rats, clearly shows that the two simple-sugars studied, fructose and glucose, elicit different types of adiposopathy (Figure 6).

Adiposopathy is characterized by ectopic fat deposition, increased visceral fat, insulin resistance, adipokine dysregulation and increased inflammatory markers [35]. Of these metabolic characteristics, neither glucose nor fructose elicited ectopic liver fat deposition or inflammation; while both sugars induced a marked increases in plasma NEFA. Only the fructose-supplemented animals showed clear, significant hyperleptinemia, increased body weight due to hypertrophy of visceral adipose tissue, and, as we have shown previously [15], insulin resistance with mild cognitive impairment. Furthermore, as it has been shown that leptin attenuates the direct insulin-sensitizing effects of adiponectin in skeletal myotubes [36], our previous $[14,15]$ and present results indicate that, similarly to what is observed over short periods of liquid fructose-supplementation [9], impaired insulin signaling in skeletal muscle is probably the main cause of the altered glucose tolerance and reduced whole-body insulin sensitivity presented by liquid-fructose-supplemented rats. Surprisingly, while glucose-supplemented rats showed no significant changes in the parameters mentioned above, and presented elevated plasma adiponectin concentrations, some inflammatory markers were increased in their vWAT samples, indicating a shift to an unhealthy, un-expandable adipose tissue phenotype. Thus, in summary, our studies clearly indicate that not all simple-sugar calories are "metabolically" equal, as those provided by fructose impose a greater burden on metabolic homeostasis than those provided by glucose. Furthermore, the unhealthy effects of fructose seem to affect primarily hypothalamus and skeletal muscle tissues, as the expansion of vWAT after chronic supplementation does not show increases in lipolytic or inflammatory activities, while allowing the accommodation of excess fat that was previously present in liver tissue.

\section{References}

[1] DiNicolantonio, J.J., Lucan, S.C., O'Keefe, J.H., The Evidence for Saturated Fat and for Sugar Related to Coronary Heart Disease. Prog. Cardiovasc. Dis. 2016, 58, 464-472.

[2] Choo, V.L., Ha, V., Sievenpiper, J.L., Sugars and obesity: Is it the sugars or the calories? Nutr. Bull. 2015, 40, 88-96.

[3] Stanhope, K.L., Sugar consumption, metabolic disease and obesity: The state of the controversy. Crit. Rev. Clin. Lab. Sci. 2015, 8363, 1-16.

[4] Stanhope, K.L., Schwarz, J.M., Keim, N.L., Griffen, S.C., Bremer, A.A., Graham, G.L., Hatcher, B., Cox, C.L., Dyachenko, A., Zhang, W., McGahan, J.P., Seibert, A., Krauss, R.M., Chiu, S., Schaefer, E.J., Ai, M., Okotozawa, S., Nakajima, K., Nakano, T., Beysen, C., Hellerstein, M.K., Berglund, L., Havel, P.J., Consuming fructose-sweetened , not glucose- sweetened, beverages increases visceral adiposity and lipids and decreases insulin sensitivity in overweight / obese humans. J. Clin. Invest. 2009, 119, 1322-1334.

[5] Rebollo, A., Roglans, N., Alegret, M., Laguna, J.C., Way back for fructose and liver metabolism: Bench side to molecular insights. World J. Gastroenterol. 2012, 18, 6552-6559. 
[6] Sangüesa, G., Shaligram, S., Akther, F., Roglans, N., Laguna, J.C., Rahimian, R., Alegret, M., Type of supplemented simple sugar, not merely calorie intake, determines adverse effects on metabolism and aortic function in female rats. Am. J. Physiol. - Hear. Circ. Physiol. 2017, 312, H289-H304.

[7] Roglans, N., Vilà, L., Farré, M., Alegret, M., Sánchez, R.M., Vázquez-Carrera, M., Laguna, J.C., Impairment of hepatic Stat-3 activation and reduction of PPARalpha activity in fructose-fed rats. Hepatology 2007, 45, 778-88.

[8] Baena, M., Sangüesa, G., Hutter, N., Sánchez, R.M., Roglans, N., Laguna, J.C., Alegret, M., Fructose supplementation impairs rat liver autophagy through mTORC activation without inducing endoplasmic reticulum stress. Biochim. Biophys. Acta 2015, 1851, 107-16.

[9] Baena, M., Sangüesa, G., Dávalos, A., Latasa, M.-J., Sala-Vila, A., Sánchez, R.M., Roglans, N., Laguna, J.C., Alegret, M., Fructose, but not glucose, impairs insulin signaling in the three major insulin-sensitive tissues. Sci. Rep. 2016, 6, 26149.

[10] Vilà, L., Roglans, N., Alegret, M., Sánchez, R.M., Vázquez-Carrera, M., Laguna, J.C., Suppressor of cytokine signaling-3 (SOCS-3) and a deficit of serine/threonine (Ser/Thr) phosphoproteins involved in leptin transduction mediate the effect of Fructose on rat liver lipid metabolism. Hepatology 2008, 48, 1506-1516.

[11] Rebollo, A., Roglans, N., Baena, M., Sánchez, R.M., Merlos, M., Alegret, M., Laguna, J.C., Liquid fructose downregulates Sirt1 expression and activity and impairs the oxidation of fatty acids in rat and human liver cells. Biochim. Biophys. Acta-Mol. Cell Biol. Lipids 2014, 1841, $514-524$

[12] Rebollo, A., Roglans, N., Baena, M., Padrosa, A., Sánchez, R.M., Merlos, M., Alegret, M., Laguna, J.C., Liquid fructose down-regulates liver insulin receptor substrate 2 and gluconeogenic enzymes by modifying nutrient sensing factors in rats. J. Nutr. Biochem. 2014, 25, 250-8.

[13] Sengupta, P., The laboratory rat: relating age with human's. Int. J. Prev. Med. 2013, 4, 624-630.

[14] Sangüesa, G., Montañés, J.C., Baena, M., Sánchez, R.M., Roglans, N., Alegret, M., Laguna, J.C., Chronic fructose intake does not induce liver steatosis and inflammation in female SpragueDawley rats, but causes hypertriglyceridemia related to decreased VLDL receptor expression. Eur. J. Nutr. 2018, https://doi.org/10.1007/s00394-018-1654-9.

[15] Sangüesa, G., Cascales, M., Griñán, C., Sánchez, R.M., Roglans, N., Pallàs, M., Laguna, J.C., Alegret, M., Impairment of Novel Object Recognition Memory and Brain Insulin Signaling in Fructose- but Not Glucose-Drinking Female Rats. Mol. Neurobiol. 2018, 55, 6984-6999.

[16] Tappy, L., Le, K.-A., Metabolic Effects of Fructose and the Worldwide Increase in Obesity. Physiol. Rev. 2010, 90, 23-46.

[17] Parlee, S.D., Lentz, S.I., Mori, H., Macdougald, O.A., Quantifying size and number of adipocytes in adipose tissue. Methods Enzymol. 2014, 537, 93-122.

[18] Bradford, M., A rapid and sensitive method for quantitation of microgram quantities of proteing utilizing the principle of protein-dye binding. Anal. Biochem. 1976, 72, 248-254.

[19] Finucane, F.M., Luan, J., Wareham, N.J., Sharp, S.J., O'Rahilly, S., Balkau, B., Flyvbjerg, A., Walker, M., Hojlund, K., Nolan, J.J., (on behalf of the European Group for the Study of Insulin Resistance: Relationship between Insulin Sensitivity and Cardiovascular Disease Risk Study Group), Savage, D.B., Correlation of the leptin: Adiponectin ratio with measures of insulin resistance in non-diabetic individuals. Diabetologia 2009, 52, 2345-2349.

[20] Miyamoto, L., Ebihara, K., Kusakabe, T., Aotani, D., Yamamoto-Takaoka, S., Sakai, T., AizawaAbe, M., Yamamoto, Y., Fukijura, F., Hayashi, T., Hosoda, K., Nakao, K., Leptin activates hepatic 5'-AMP-activated protein kinase through sympathetic nervous system and $\alpha 1$-adrenergic receptor: A potential mechanism for improvement of fatty liver in lipodystrophy by leptin. J. Biol. Chem. 2012, 287, 40441-40447. 
[21] Yamauchi, T., Nio, Y., Maki, T., Kobayashi, M., Takazawa, T., Iwabu, M., Okada-Iwabu, M., Kawamoto, S., Kubota, N., Kubota, T., Ito, Y., Kamon, J., Tsuchida, A., Kumagai, K., Kozono, H., Hada, Y., Ogata, H., Tokuyama, K., Tsunoda, M., Ide, T., Murakami, K., Awazawa, M., Takamoto, I., Froguel, P., Hara, K., Tobe, K., Nagai, R., Ueki, K., Kadowaki, T., Targeted disruption of AdipoR1 and AdipoR2 causes abrogation of adiponectin binding and metabolic actions. Nat. Med. 2007, 13, 332-339.

[22] Sáinz, N., Barrenetxe, J., Moreno-Aliaga, M.J., Martínez, J.A., Leptin resistance and diet-induced obesity: Central and peripheral actions of leptin. Metabolism. 2015, 64, 35-46.

[23] Oswal, A., Yeo, G., Leptin and the control of body weight: A review of its diverse central targets, signaling mechanisms, and role in the pathogenesis of obesity. Obesity 2010, 18, 221-229.

[24] Mantzoros, C.S., Magkos, F., Brinkoetter, M., Sienkiewicz, E., Dardeno, T.A., Kim, S-Y., Hamnvik, O-P.R., Koniaris, A., Leptin in human physiology and pathophysiology. AJP Endocrinol. Metab. 2011, 301, E567-E584.

[25] Banks, W.A., Coon, A.B., Robinson, S.M., Moinuddin, A., Shultz, J.M., Nakaoke, R., Morley, J.E., Triglycerides Induce Leptin Resistance at the Blood-Brain Barrier. Diabetes 2004, 53, $1253-$ 1260 .

[26] Banks, W.A., Farr, S.A., Salameh, T.S., Niehoff, M.L., Rhea, E.M., Morley, J.E., Hanson, A.J., Hansen, K.M., Craft, S., Triglycerides cross the blood-brain barrier and induce central leptin and insulin receptor resistance. Int. J. Obes. 2018, 42, 391-397.

[27] Reed, A.S., Unger, E.K., Olofsson, L.E., Piper, M.L., Myers Jr., M.G., Xu, A.W, Functional Role of Suppressor of Cytokine Signaling 3 Long-Term Energy Homeostasis. Diabetes 2010, 59, 894906.

[28] Howard, J.K., Flier, J.S., Attenuation of leptin and insulin signaling by SOCS proteins. Trends Endocrinol. Metab. 2006, 17, 365-371.

[29] Münzberg, H., Morrison, C.D., Structure, production and signaling of leptin. Metabolism. 2015, $64,13-23$.

[30] Cohen, P., Yang, G., Yu, X., Soukas, A.A., Wolfish, C.S., Friedman, J.M., Li, C., Induction of Leptin Receptor Expression in the Liver by Leptin and Food Deprivation. J. Biol. Chem. 2005, 280, 10034-10039.

[31] St-Pierre, J., Tremblay, M.L., Modulation of leptin resistance by protein tyrosine phosphatases. Cell Metab. 2012, 15, 292-297.

[32] Stern, J.H., Rutkowski, J.M., Scherer, P.E., Adiponectin, Leptin, and Fatty Acids in the Maintenance of Metabolic Homeostasis through Adipose Tissue Crosstalk. Cell Metab. 2016, 23, $770-784$.

[33] Bell, B.B., Harlan, S.M., Morgan, D.A., Guo, D.F., Rahmouni, K., Differential contribution of POMC and AgRP neurons to the regulation of regional autonomic nerve activity by leptin. Mol. Metab. 2018, 8, 1-12.

[34] Rezvani, R., Cianflone, K., McGahan, J.P., Berglund, L., Bremer, A.A., Keim, N.L., Griffen, S.C., Havel, P.J., Stanhope, K.L., Effects of sugar-sweetened beverages on plasma acylation stimulating protein, leptin and adiponectin: Relationships with Metabolic Outcomes. Obesity 2013, 21, 2471-2480.

[35] Neeland, I.J., Poirier, P., Després, J.-P., Cardiovascular and Metabolic Heterogeneity of Obesity. Circulation 2018, 137, 1391-1406.

[36] Fang, X., Fetros, J., Dadson, K.E., Xu, A., Sweeny, G., Leptin prevents the metabolic effects of adiponectin in L6 myotubes. Diabetologia 2009, 52, 2190-2200.

\section{Author contributions}


G.S performed the experiments and analysed the data. M.B and A.M.V ran the animal study and contributed to PCR experiments. J.C.M. performed vWAT analysis. R.M.S helped in data interpretation and reviewed the manuscript. N.R contributed to western blot experiments and prepared the figures. J.C.L and M.A designed the experiments, supervised data analysis and wrote the manuscript.

\section{Acknowledgements}

This work was supported by grants from the Fundació Privada Catalana de Nutrició i Lípids, the Spanish Ministry of Economy, Industry and Competitiveness [SAF2013-42982-R and SAF2017-82369-R] and the European Regional Development Fund (ERDF). Gemma Sangüesa and Miguel Baena were supported by FPU and FPI grants, respectively, from the Spanish Ministry of Science and Innovation. Ana Magdalena Velazquez is a pre-doctoral fellow, BECAL grant program BCAL04-327, from the Government of Paraguay. We are recognized as a Consolidated Research Group by the Autonomous Government of Catalonia (2017 SGR 38). We would like to thank the University of Barcelona's Language Services for revising the manuscript.

\section{Conflict of interest}

All authors approved the final version of the manuscript and have no conflict of interest. 


\section{Figure Legends}

Figure 1. Plasma leptin concentrations (A) from CT (control female Sprague-Dawley -SD- rats), GLC (female SD rats supplemented with a glucose solution prepared to match the number of calories ingested by the fructose group) and FRC (female SD rats supplemented with a $10 \% \mathrm{w} / \mathrm{v}$ fructose solution as drinking water for seven months) experimental groups; bar plots represent the mean \pm S.D for each group. (B) Western-blot of SOCS3 protein in liver samples obtained from the three experimental dietary groups; bar plots represent the mean \pm S.D. band intensity of 4-5 different samples for each group. Representative bands shown in the upper part of the figure correspond to 3 different rats in each group. Western-blot of phosphor- and total-STAT3 (C), and phosphor- and total-AMPK (D) proteins, respectively, in liver samples obtained from the three experimental dietary groups; bar plots the mean \pm S.D band intensity of the phosphor- or total-proteins of 4-5 different samples for each group. Representative bands shown in the upper part of the figure correspond to 3 different rats in each group. $* \mathrm{P}<0.05, * * \mathrm{P}<0.01$ vs CT values.

Figure 2. (A) Bar plots representing the mean \pm S.D. mRNA levels corresponding to the socs 3 gene in hypothalamus, frontal cortex and hippocampus tissue samples obtained from CT (control female SpragueDawley -SD- rats, $n=6$ ), GLC (female SD rats supplemented with a glucose solution prepared to match the number of calories ingested by the fructose group, $\mathrm{n}=8$ ), and FRC (female SD rats supplemented with a $10 \% \mathrm{w} / \mathrm{v}$ fructose solution as drinking water for seven months, $\mathrm{n}=8$ ) experimental groups. (B) Bar plots representing the mean \pm S.D. mRNA levels corresponding to agrp, npy, cart, pomc and obrb hypothalamus genes from CT $(n=6)$, GLC $(n=8)$, and FRC $(n=8)$ experimental groups. (C) Western-blot of phosphorand total-AMPK, and phosphor- and total ERK 1/2 proteins in hypothalamus samples obtained of 4-5 different samples from the three experimental dietary groups. Representative bands correspond to 3 different rats in each group. ${ }^{*} \mathrm{P}<0.05, * * \mathrm{P}<0.01$ vs $\mathrm{CT}$ values.

Figure 3. Bar plots representing the mean \pm S.D. plasma NEFA concentrations (A), plasma NEFA concentrations relative to the ratio vWAT weight/femur length $(\mathbf{B})$, and the ratio vWAT weight/femur length (C) in CT (control female Sprague-Dawley -SD- rats), GLC (female SD rats supplemented with a glucose solution prepared to match the number of calories ingested by the fructose group) and FRC (female SD rats supplemented with a $10 \% \mathrm{w} / \mathrm{v}$ fructose solution as drinking water for seven months) experimental groups. (D) Western-blot of phosphor- and total-Akt proteins, respectively, in vWAT samples obtained from the three experimental dietary groups; bar plots the mean \pm S.D band intensity of the phosphor-Akt protein of 4-5 different samples for each experimental group. Representative bands shown in the upper part of the figure correspond to 3 different rats in each experimental group. (E) Bar plots representing the mean \pm S.D. plasma insulin concentrations in the three experimental groups. (F) Bar plots representing the mean \pm S.D. mRNA levels corresponding to fasn and $s c d 1 \mathrm{vWAT}$ genes from CT $(\mathrm{n}=6), \mathrm{GLC}(\mathrm{n}=8)$, and FRC $(\mathrm{n}=8)$ experimental groups. $* \mathrm{P}<0.05, * * \mathrm{P}<0.01$ vs CT values.

Figure 4. (A) Bar plots representing the mean \pm S.D. mRNA levels corresponding to the socs 3 gene in vWAT tissue samples obtained from CT (control female Sprague-Dawley -SD- rats, n=6), GLC (female SD rats supplemented with a glucose solution prepared to match the number of calories ingested by the fructose group, $\mathrm{n}=8$ ), and FRC (female SD rats supplemented with a $10 \% \mathrm{w} / \mathrm{v}$ fructose solution as drinking water for seven months, $n=8$ ) experimental groups. (B) Western-blot of ATGL protein in vWAT obtained of 4-5 different samples from the three experimental dietary groups; bar plots represent the mean \pm S.D. band intensity for each group. Representative bands shown in the upper part of the figure correspond to 3 different rats in each group. (C) Representative hematoxylin and eosin vWAT sections from CT, GLC, and FRC experimental groups. (D) Bar plots representing the mean percent frequency distribution of adipocyte area in the three experimental groups. (E) Bar plots representing the AUC frequency mean \pm S.D. percentage of adipocytes with a surface area $<$ or $\geq$ to $8,000 \mu \mathrm{m}^{2}$ in the three experimental groups. ${ }^{\#} \mathrm{P}=0.08 ;{ }^{*} \mathrm{P}<0.05$ vs $\mathrm{CT}$ values.

Figure 5. (A) Bar plots representing the mean \pm S.D. mRNA vWAT levels corresponding to the genes $o b$ and adipoq from CT (control female Sprague-Dawley -SD- rats, $\mathrm{n}=6$ ), GLC (female SD rats supplemented with a glucose solution prepared to match the number of calories ingested by the fructose 
group, $\mathrm{n}=8$ ), and FRC (female SD rats supplemented with a $10 \% \mathrm{w} / \mathrm{v}$ fructose solution as drinking water for seven months, $n=8$ ) experimental groups. (B) Bar plots representing the mean \pm S.D. of the ratio between lepin and adiponectin plasma concentrations in the three experimental groups. (C) Western-blot of PKAc, and phosphor- and total-HSL proteins, respectively, in vWAT obtained of 4-5 different samples from CT, GLC, and FRC groups. Representative bands shown correspond to 3 different rats in each group. ${ }^{*} \mathrm{P}=0.08 ;{ }^{*} \mathrm{P}<0.05$ vs $\mathrm{CT}$ values.

Figure 6. Schematic overview of the differential adiposopathy induced by chronic liquid glucoseand fructose-supplementation in female Sprague-Dawley rats. Adiposopathy is characterized by ectopic fat deposition, increased visceral fat, insulin resistance, adipokine dysregulation and increased inflammatory markers. Of these metabolic signatures, neither glucose nor fructose elicited ectopic liver fat deposition and inflammation, while both sugar induced marked increases in plasma NEFA. Only fructose-supplemented animals showed clear, significant hyperleptinemia, insulin resistance with mild cognitive impairment and increased body weight due to a hypertrophic process of visceral adipose tissue. Glucose-supplemented rats showed no significant changes in the above mentioned parameters and presented elevated plasma adiponectin concentrations, some inflammatory markers were increased in their vWAT samples, indicating a shift to an unhealthy, un-expandable adipose tissue phenotype. We have used data from our previous $[14,15]$ and present work. 
A
B
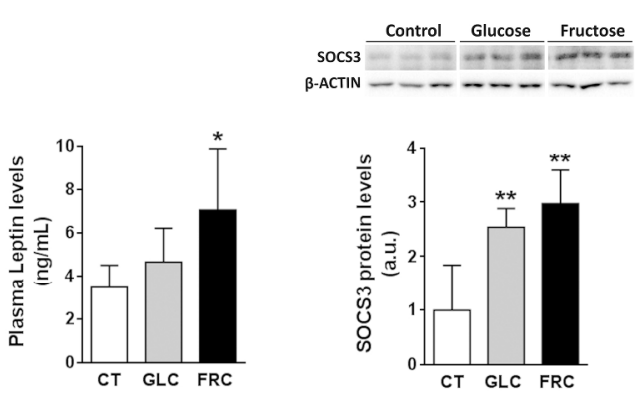

C

D
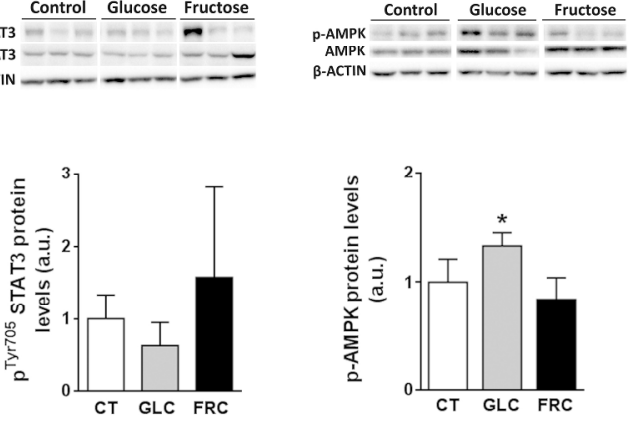

Figure 1

$250 \times 297 \mathrm{~mm}(300 \times 300$ DPI $)$ 
A

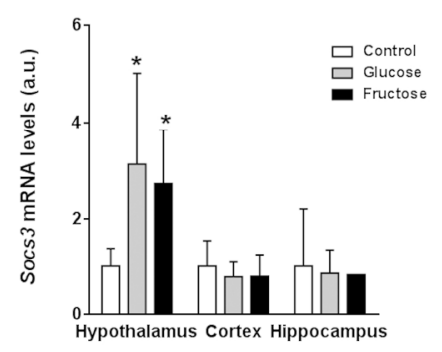

B

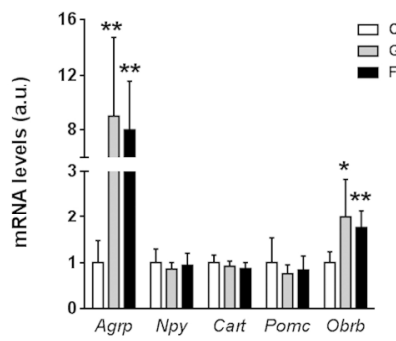

C

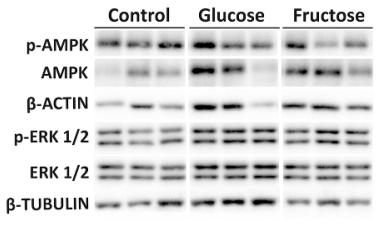

Figure 2

$250 \times 297 \mathrm{~mm}(300 \times 300$ DPI $)$

Wiley-VCH 
A

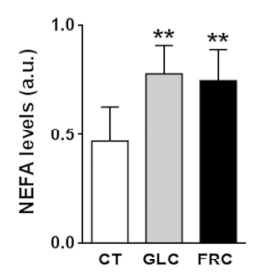

D
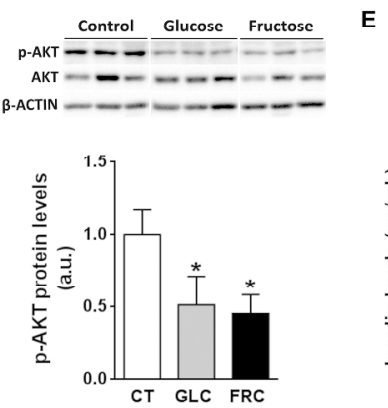

B
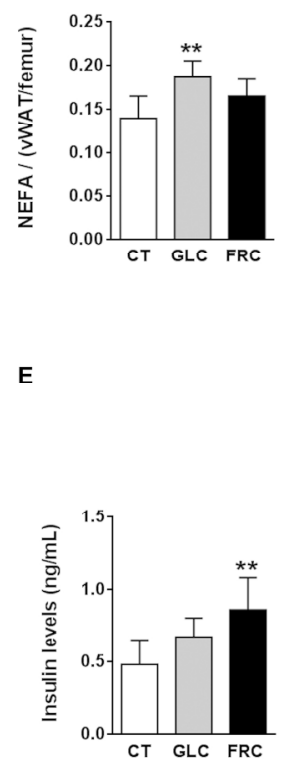

C

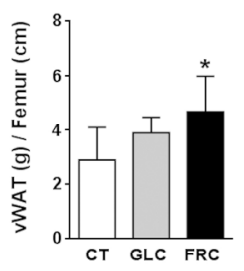

$\mathbf{F}$

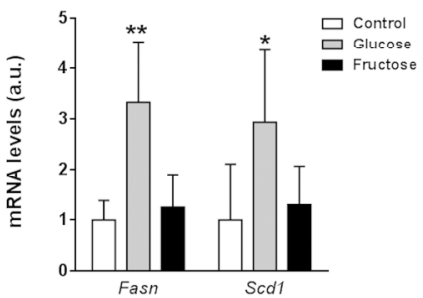

Figure 3

$250 \times 297 \mathrm{~mm}(300 \times 300$ DPI $)$

Wiley-VCH 
A
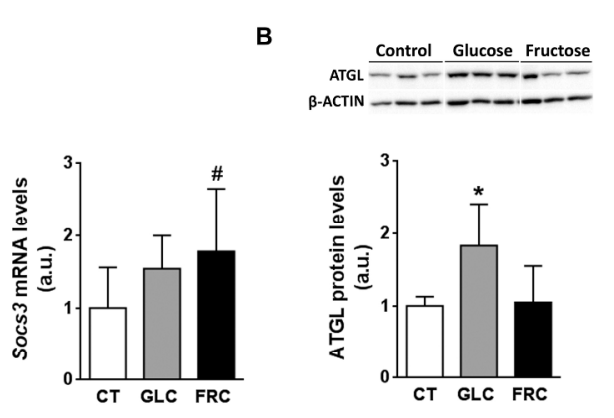

C

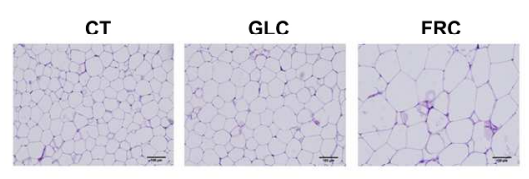

D

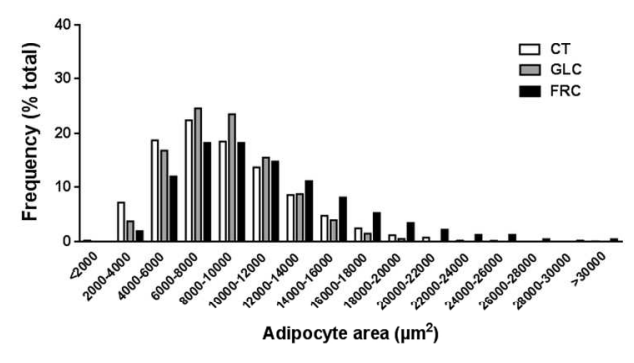

E

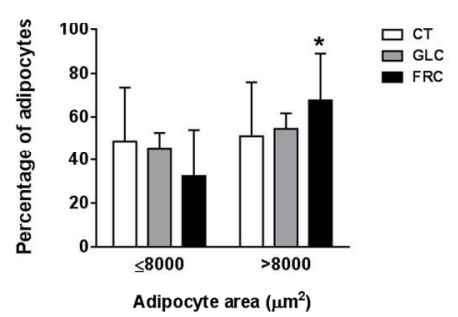

Figure 4

$250 \times 297 \mathrm{~mm}(300 \times 300 \mathrm{DPI})$ 
A

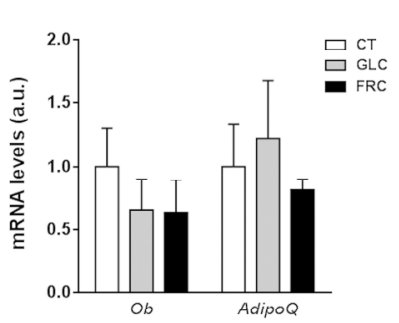

C

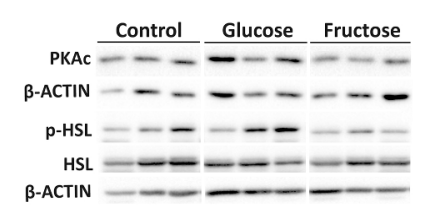

B

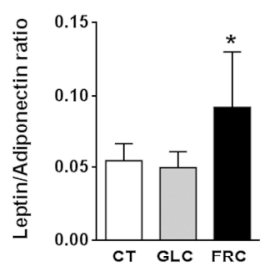

D

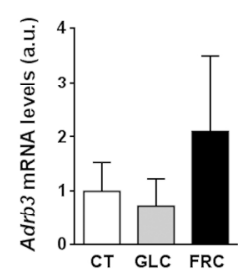

Figure 5

$250 \times 297 \mathrm{~mm}(300 \times 300$ DPI $)$

Wiley-VCH 


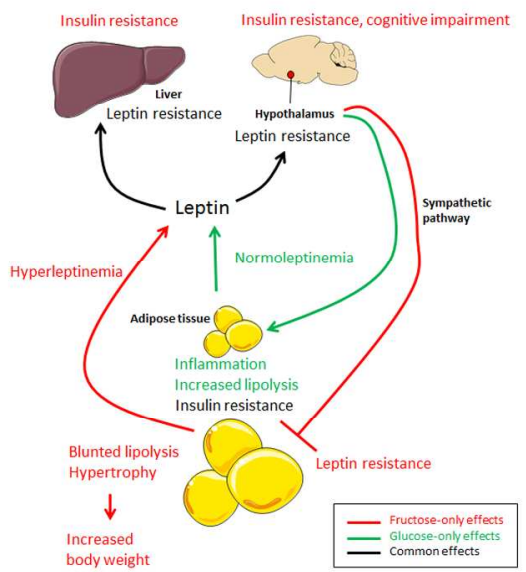

Figure 6 $209 \times 297 \mathrm{~mm}(300 \times 300 \mathrm{DPI})$

Wiley-VCH 